\title{
Detection of Cochlear Hearing Loss Applying Wavelet Packets and Support Vector Machines
}

\author{
Hubert Dietl ${ }^{1}$, Stephan Weiss ${ }^{1}$ \\ ${ }^{1}$ Dept. Electronics \& Computer Science, University of Southampton, UK \\ hwd, s.weiss@ecs. soton.ac.uk
}

\begin{abstract}
The aim of this paper is to evaluate the application of the wavelet packet transform (WP) and support vector machines (SVM) to transient evoked otoacoustic emissions (TEOAE) in order to achieve a detection of frequency-specific hearing loss. We introduce a system to determine detection rates between groups of persons with normal hearing, high frequency hearing loss, and pantonal hearing loss. The validity and use of our approach is verified on a different patient group.
\end{abstract}

\section{INTRODUCTION}

Transient evoked otoacoustic emissions (TEOAE) are used as a clinical standard procedure to detect cochlear hearing loss [1], and measurement equipment [2] is widely available in hospitals. The analysis of TEOAE is usually performed by an human expert. Recently, signal processing detection systems aiming at an automated detection of cochlear hearing loss have been motivated to assist or replace the human expert. These studies aiming at detection of TEOAE apply discrete wavelet transform and neural networks [3],[4]. Here, we introduce a system applying a WP for feature extraction, a signal-to-noise (SNR)-like criterion for feature selection and support vector machines for classification.

Fig. 1 gives an overview of our system. For the feature extraction, a WP is applied. To select the features of the data, an SNR-like criterion is applied to the transformed data resulting in a reduction of coefficients to be used for classification and aiming at a reduction of noisy coefficients. This approach will be outlined in more detail in Sec. 3, following a de- scription of TEOAE data in Sec. 2. The classification of the data is conducted by a support vector machine (SVM) classifier explained in Sec. 4 more explicitly. In Sec. 5, based on the training data, a support vector classification network is found and applied to the test data group yielding detection rates which describe the performance of the system and can be compared with other studies. Finally, Sec. 6 draws the conclusions.

\section{TEOAE AND WAVELET PACKET TRANSFORM}

The patient data consists of two sets measured at the Universities of Homburg and Heidelberg, with each consisting of an evaluation of more than 200 ears. The Homburg data represents the training data, the Heidelberg data is addressed as test data. Both sets are classified to one of the three groups of normal hearing $(\mathrm{NH})$, pantonal (PT), or high frequency (HF) hearing loss, as defined in Fig. 2. For each ear, the TEOAE equipment measured a total of 520 responses, each for a period of $20.48 \mathrm{~ms}$, and calculated two partial averages (labelled A and B) alternatingly over 260 responses each.

Due to the transient nature of the signals, previous work on the qualitative analysis of TEOAE has focused on time-frequency (TF) methods, such as filter banks [5], matching pursuit [6], or discrete wavelet transforms (DWT) [3], whereby a quantitative study w.r.t. the achievable distinction of frequency-specific hearing loss has been performed in [3], based on the DWT.

The wavelet packet transform (WP) can be seen as a more general transform compared to the DWT. Therefore, we briefly describe the DWT here. The DWT is

\begin{tabular}{|c|c|c|c|c|c|}
\hline Training data & Feature extraction: & TF coefficients for training & Feature selection: & Classification: & - Trained SVM classifier \\
\hline Test data & Wavelet Packet transform & TF coefficients for test & $\begin{array}{l}\text { Selection of coefficients } \\
\text { by SNR-like criterion }\end{array}$ & Support vector machines & $\longrightarrow$ Detection rates for test data \\
\hline
\end{tabular}

Fig. 1. Overview of the detection system for cochlear hearing loss. 

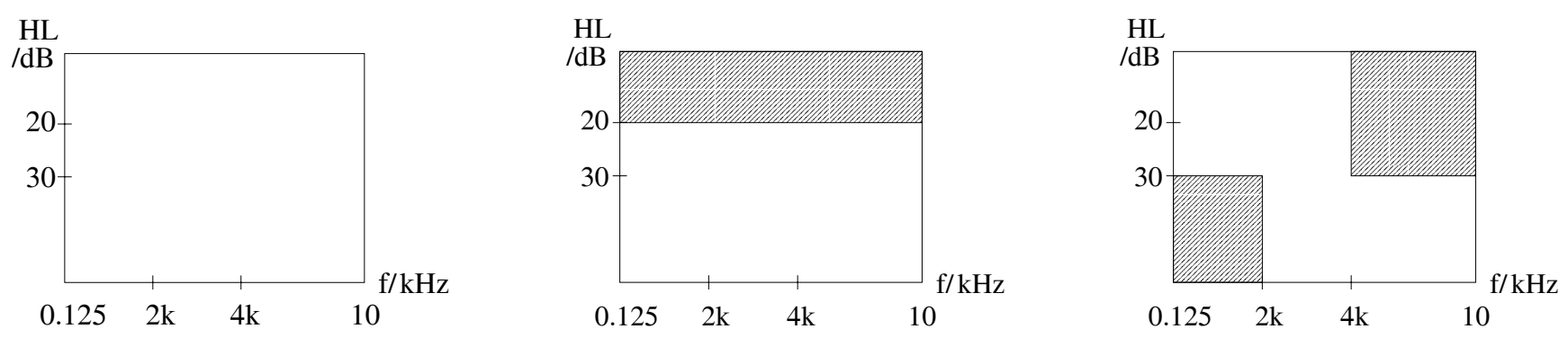

Fig. 2. Characterisation of hearing loss for (left) normal hearing, (middle) pantonal HL, and (right) high frequency $\mathrm{HL}$.

a fixed transform based on a "mother wavelet" from which the transformation coefficients are derived by scaling, translation and sampling. Here, we have chosen the Mallat wavelet for which good results have been reported in similar studies [3]. The transform coefficients approximately cover TF tiles as illustrated in Fig. 3 a).

The WP transform is an adaptive transformation similar to the DWT but with a flexible partitioning of the TF plane. The advantage of this approach compared to the DWT is that the entropy of the transformed data shall be minimised through variable levels of decomposition such that the energy is concentrated in as few coefficients as possible. That minimisation is achieved by the reduction of the concentration according to Shannon's entropy [7]. Fig. 3 b) shows a sample WP decomposition.

Based on a parameterisation of the data by the WP, representing the feature extraction of the data, the application of an SNR-like criterion for the feature selection is conducted which will be described next.

\section{FEATURE SELECTION}

To quantify and exploit differences in the TEOAE WP coefficients of the three groups of hearing ability within the Homburg data, a signal-to-noise-ratio (SNR) based criterion is invoked. First, the SNR is estimated for each of the 512 parameters in the TF-plane based on the WPs of the two partial averages, $\mathrm{WP}_{\mathrm{A}}(n)$ and $\mathrm{WP}_{\mathrm{B}}(n), n=1, \ldots, 512$. The SNR of the $n$th coefficient is (coarsely) estimated by comparing the sum and the difference obtained from the partial averages $\mathrm{A}$ and $\mathrm{B}$ :

$$
\operatorname{SNR}(n)=20 \log _{10} \frac{\left|\mathrm{WP}_{\mathbf{A}}(n)+\mathrm{WP}_{\mathbf{B}}(n)\right|}{\left|\mathrm{WP}_{\mathbf{A}}(n)-\mathrm{WP}_{\mathbf{B}}(n)\right|+\epsilon} .
$$

This SNR is calculated for all measurements, and for each of the $512 \mathrm{WP}$ coefficients within each of the three hearing ability groups, the distribution is recorded.
The SNR value of a WP coefficient is used to evaluate the separability of any two groups with different hearing status. The separability can be assessed independent of the selection of a specific threshold by means of a socalled receiver operating characteristic (ROC) curve. The area underneath the ROC is a measure for the separability of both groups, and independent of the definition of SNR-thresholds [8].

As single WP coefficients yield a poor separability between any two groups, we pick the coefficient that gives the best separable SNR according to (1) as a starting value and iteratively grow a coefficient set $\mathcal{G}$ to improve separability. Further coefficients are added to $\mathcal{G}$ from the neighbourhood of surrounding coefficients. Adjacency is defined by edge and corner connections in the TF plane. The iteration is stopped when the ROC does not further improve for the SNR of the coefficients contained in $\mathcal{G}$.

\section{SVM CLASSIFICATION}

In the following, we briefly explain SVM, [9],[10]. We consider a three class classification problem for the classes defined by the groups NH, HF and PT, starting with an explanation for a two class classification. The training data originates from the Homburg data, while the test data comprises the Heidelberg measurements.

The training data is described as a set of training vectors $\left\{\mathbf{p}_{i}\right\}_{i=1} \ldots M$ with corresponding binary labels $S_{i}=1$ for the one class, e.g. NH, and $S_{i}=-1$ for the second class, e.g. HF. The SVM conducts a classification of a test vector $\mathbf{t}$ by assigning a label $\hat{S}$ by calculating

$$
\hat{S}=\operatorname{sign}(f(\mathbf{t})) \quad \text { with } \quad f(\mathbf{t})=\sum_{i} \alpha_{i} S_{i} K\left(\mathbf{t}, \mathbf{p}_{i}\right)+b .
$$

The $\alpha_{i}$ are called weights and $b$ is the bias, which are SVM parameters and adopted during training by max- 

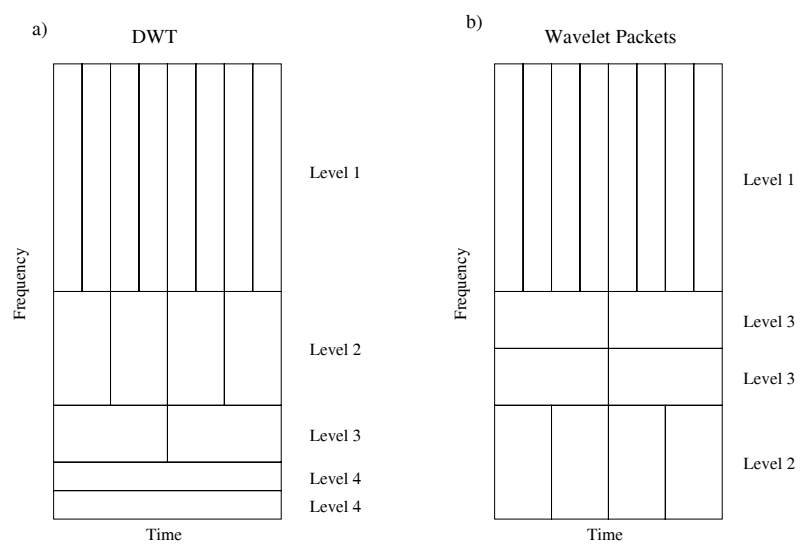

Fig. 3. TF tiling comparison between a) a DWT and b) a sample WP decomposition.

imising

$$
L_{D}=\sum_{i} \alpha_{i}-\frac{1}{2} \sum_{i, j} \alpha_{i} \alpha_{j} S_{i} S_{j} K\left(\mathbf{p}_{i}, \mathbf{p}_{j}\right)
$$

under the constraints

$$
0 \leq \alpha_{i} \leq C \quad \text { and } \quad \sum_{i} \alpha_{i} S_{i}=0
$$

with $C$ being a positive constant which weighs the influence of training errors. $K(\cdot, \cdot)$ is called kernel of the SVM. If there is a solution for $\alpha_{i}$, a value for $b$ is determined. Usually $\alpha_{i}=0$ for the majority of $i$ and thus the summation in (2) is limited to a subnet of the $\mathbf{p}_{i}$, which therefore is called the set of support vectors. There are several commonly used kernels for SVM, which give some flexibility for the underlying application. Many implementations of kernels can be found in literature, whereby two popular ones are Gaussian and polynomial kernels. If $K(\cdot, \cdot)$ is positive definite, (3) and (4) is a convex quadratic optimisation problem, which converges towards the global optimum assuringly. This optimisation can be quite demanding in terms of computation time for real-world problems, and therefore, sophisticated algorithms like sequential minimal optimisation (SMO) [9] are used for the solution.

To find a significant value for the training error $C$, a leave-one-out (1-o-o) estimation of the error rate is applied as follows: From the training samples, remove the first example. Train the SVM on the remaining samples. Then test the removed example. If the example is classified incorrectly, it is said to produce a leave-one-out error. In [9], an approach to estimate the maximum l-o-o error is shown avoiding training the SVM more than once, which is also used for our study. By changing the value for $C$ stepwise, the minimum for the l-o-o error is found determining the SVM classification network. For our application, a Gaussian kernel was used.

So far, we have described the SVM for only two classes. As we aim at distinguishing 3, we need to define a multi-class method. In [11] a decision directed acyclic graph (DAG) for multi-class SVM is introduced. It is based on an 1-vs-1 classification where the training is conducted for all possible combinations of the classes. Based on a trained SVM classifier for each possible class combination, a binary acyclic graph is used for testing. Fig. 4 shows the decision DAGSVM for our application to the the three classes with different hearing ability.

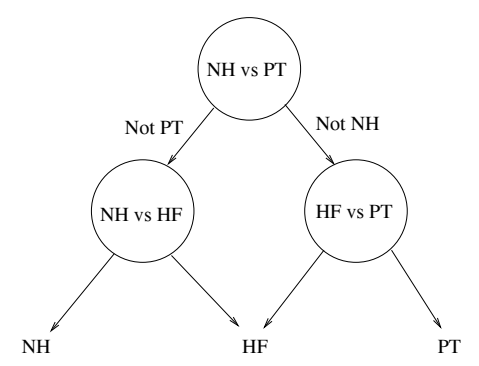

Fig. 4. DAGSVM for TEOAE.

\section{RESULTS AND DISCUSSION}

Having described the detection methods and the data used for our system, we present the results in the following. Fig. 5 illustrates the average WP coefficient energy for the training data showing the typical TEOAE properties of high frequencies occurring early and low frequencies appearing late [6]. In Figure 6 the isolated coefficients for each distinction case are shown. They were found by the search procedure based on the SNR criterion explained in Sec. 3 and appear to be reasonably located when compared to the average energies in Fig. 5.

Based on the coefficient sets, a SVM classification is conducted for each distinction case using the training data. The test data is analysed by the determined classifiers according to the decision DAG in Fig. 4 yielding the detection rates in Tab. 1 for each class.

The table shows that the HF can be detected more significantly than the NH group with the explained system. The PT group is the most difficult to determine, just above half of the patients can be allocated correctly. These results may not seem to be encouraging. However, when only considering the the case $\mathrm{NH}$ vs PT, $89.9 \%$ of the $\mathrm{NH}$ group and $84.6 \%$ of the PT group are 


\begin{tabular}{|c|c|}
\hline group & $\begin{array}{c}\text { detection rate } \\
\text { for test data }\end{array}$ \\
\hline $\mathrm{NH}$ & $68.1 \%$ \\
$\mathrm{HF}$ & $74.7 \%$ \\
$\mathrm{PT}$ & $56.4 \%$ \\
\hline
\end{tabular}

Tab. 1. Detection rates yielded by DAGSVM.

allocated correctly by the system which is well in the range of other studies.

E.g in [12], a group of normal hearing is defined by no hearing loss up to $30 \mathrm{~dB}$ and a hearing impaired group with a hearing loss over $30 \mathrm{~dB}$. A separation method based on wavelet transforms, ensemble correlation, time window design and mean cross-correlation is introduced. The study concludes that by standard analysis $90 \%$ of the normal hearing persons and $65 \%$ of the hearing impaired patients can be allocated correctly. By applying the various methods, the value for the hearing impaired group is increased by approximately $17 \%$ to $83 \%$ in that study. Compared to our study we achieve slightly better results when only considering the case $\mathrm{NH}$ vs $\mathrm{PT}$, which can be seen as equivalent to the case shown in [12]. One could also argue, that our methods lead to a better separation of hearing loss as our threshold for defining the difference between $\mathrm{NH}$ and $\mathrm{PT}$ was $20 \mathrm{~dB}$, and the worse the hearing loss gets, the weaker the TEOAE appear and therefore the easier it should be to separate them. On the other hand, we achieve the lowest value of $56 \%$ for the PT group, which shows that it is easier to separate when clear TEOAE are present, which is more likely the case for a threshold of hearing loss of $20 \mathrm{~dB}$ than for $30 \mathrm{~dB}$. Recapitulating it can be said that our approach yields separation results than can well compete with other studies so far.

\section{CONCLUSIONS}

We have presented a WP analysis of TEOAE that aims at the detection of frequency specific hearing loss. We have motivated the use of TF methods, and proposed a method to optimise a set of distinctive WP coefficients. This maximisation represents the input to a SVM classifier for the detection. We used two data sets for training and testing. The validity of the results was verified by a test group. Moreover, the obtained results proved to be competitive when they were compared to similar study which also aims at the detection of TEOAE. Therefore, the results appear reasonably robust and encourage frequency specific hearing loss detection via signal processing of TEOAE.

\section{ACKNOWLEDGEMENTS}

The authors would like to acknowledge Prof. Ulrich Hoppe and Sebastian Hoth of the University of Erlangen, Germany, who kindly provided valuable expertise and the data.

\section{REFERENCES}

[1] M.S. Robinette and T.J. Glattke, Otoacoustic Emissions: Clinical Applications, Thieme Medical Pub, 2. edition, 2001.

[2] Otodynamics Ltd., Hatfield, Herfortshire, UK, ILO OAE Instrument User Manual, 5a edition, October 1997.

[3] S. Weiss, U. Hoppe, M. Schabert, and U. Eysholdt, "Wavelet Analysis of Transient Evoked Otoacoustic Emissions for Differential Diagnosis of Cochlear Hearing Loss," in Asilomar Conference on Signals, Systems, and Computers, Monterey, CA, November 2001.

[4] G. Buller and M.E. Lutman, "Automatic classification of transiently evoked otoacoustic emissions using an artificial neural network," British Journal of Audiology, vol. 32, pp. 235-247, 1998.

[5] P. Ravazzani, G. Tognola, F. Grandori, and J. Ruohonen, "Two-Dimensional Filter to Facilitate Detection of Transient-Evoked Otoacoustic Emissions," IEEE Transactions on Biomedical Engineering, vol. 45, pp. 1089-1096, 1998.

[6] K. J. Blinowska, P. J. Durka, A. Skierski, F. Grandori, and G. Tognola, "High Resolution Time-Frequency Analysis of Otoacoustic Emissions," Technology and Health Care, vol. 5, pp. 407-418, 1997.

[7] A. Jensen and Anders la Cour Harbo, Ripples in Mathematics, Springer-Verlag, Berlin, Heidelberg, New York, 2001.

[8] J. A. Hanley and B. J. McNeil, "The Meaning and Use of the Area under a Receiver Operating Characteristic (ROC) Curve," Radiology, vol. 143, pp. 26-36, 1982.

[9] V.N. Vapnik, Statistical Learning Theory, Cambridge University Press, New York: Wiley, 1998.

[10] C. Bahlmann, B. Haasdonk, and H. Burkhardt, "Online Handwriting Recognition with Support Vector Machines - A Kernel Approach," in Proceedings of the 8th Int. Workshop on Frontiers in Handwriting Recognition (IWFHR), pp. 49-54, 2002.

[11] J. Platt, N. Cristianini, and J. Shawe-Taylor, "Large Margin DAGs for Multiclass Classification," in Proceedings of Advances in Neural Information Processing Systems, NIPS'99, MIT Press 2000, pp. 547-553.

[12] A. Janusauskas, L. Sornmo, O. Svensson, and B. Engdahl, "Detection of Transient-Evoked Otoacoustic Emissions and the Design of Time Windows," IEEE Transactions on Biomedical Engineering, vol. 49, no. No.2, pp. 132-139, February 2002. 

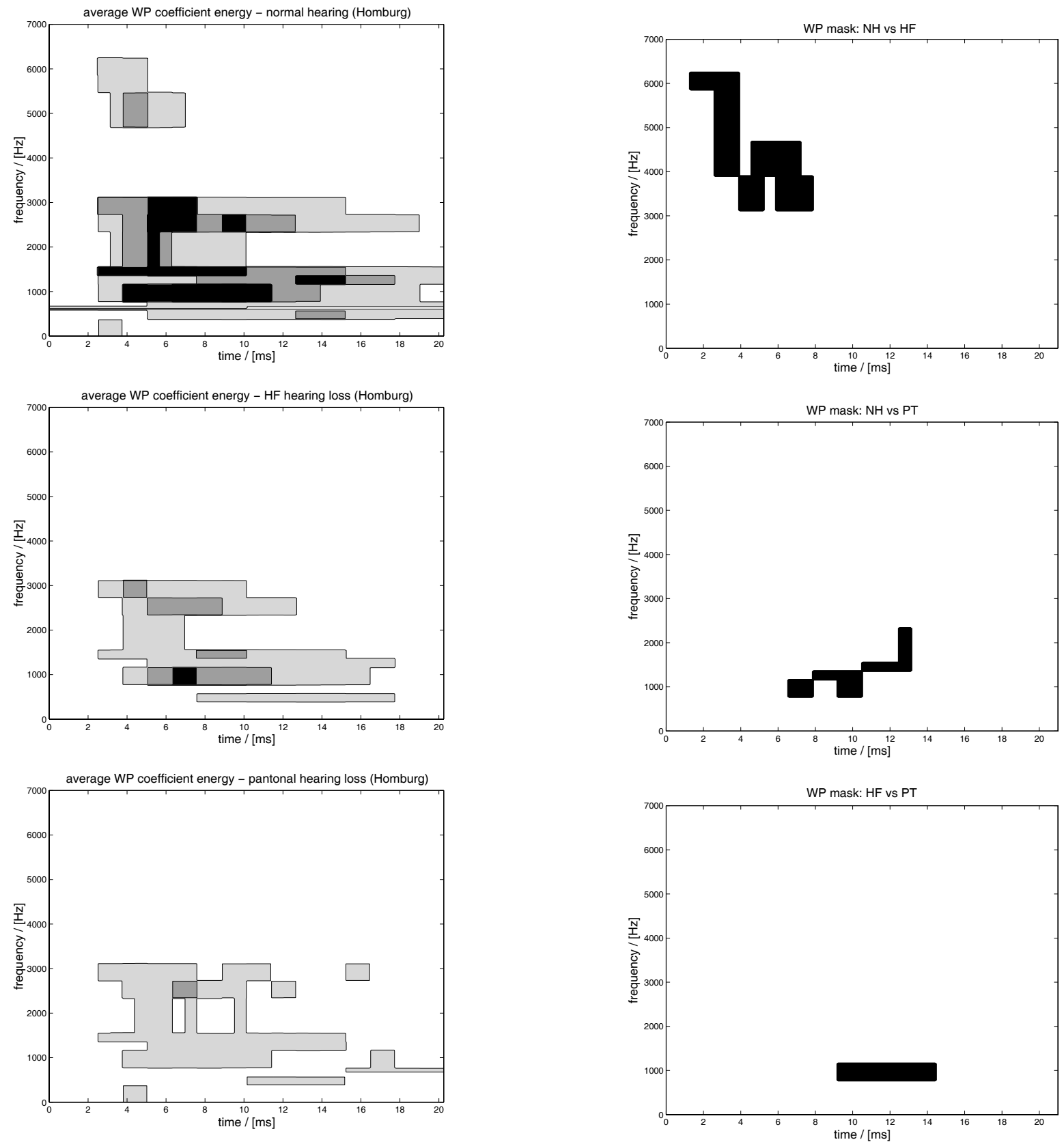

Fig. 5. Average WP coefficient energy for the different hearing ability groups for the training data.

Fig. 6. Selected feature coefficients for the three distinction cases. 